\title{
Economic evaluation of stepped-care versus usual care for depression and anxiety in older adults with vision impairment: randomized controlled trial
}

\author{
Hilde P. A. van der Aa ${ }^{1 *}$, Ger H. M. B. van Rens ${ }^{1,2}$, Judith E. Bosmans ${ }^{3}$, Hannie C. Comijs ${ }^{4}$
} and Ruth M. A. van Nispen ${ }^{1}$

\begin{abstract}
Background: A stepped-care program was found effective in preventing depressive and anxiety disorders in older adults with vision impairment. However, before a decision can be made about implementation, the costeffectiveness of this program should be investigated. Therefore, we aimed to compare the cost-effectiveness of stepped-care versus usual care within low vision rehabilitation.
\end{abstract}

Methods: An economic evaluation from a societal perspective was performed alongside a multicenter randomized controlled trial. Data were collected by masked assessors during 24 months. Included were 265 older adults with vision impairment and subthreshold depression and/or anxiety. They were randomly assigned to stepped-care plus usual care $(n=131)$ or usual care alone $(n=134)$. Stepped-care comprised 1$)$ watchful waiting, 2$)$ guided self-help based on cognitive behavioral therapy, 3) problem solving treatment, and 4) referral to a general practitioner. Costs were based on direct healthcare costs and indirect non-healthcare costs. Main outcome measures were quality-adjusted life years (QALYs) and the cumulative incidence of major depressive, dysthymic and/or anxiety disorders. Secondary outcomes were symptoms of depression and anxiety.

Results: Based on intention-to-treat, significant differences were found in the incidence of depressive/anxiety disorders (mean difference $0.17 ; 95 \% \mathrm{Cl} 0.06$ to 0.29 ) and symptoms of anxiety (mean difference $1.43,95 \% \mathrm{Cl} 0.10$ to 2.77) in favor of stepped-care versus usual care; no significant difference was found for QALYs and symptoms of depression. Societal costs were non-significantly lower in the stepped-care group compared with the usual care group (mean difference: -€877; 95\% confidence interval (Cl): -8039 to 5489). Cost-effectiveness acceptability curves showed that the probability of cost-effectiveness was $95 \%$ or more at a willingness-to-pay of $€ 33,000$ per disorder prevented. The probability that stepped-care was cost-effective compared to usual care was $59 \%$ or more for a ceiling ratio of $0 € /$ QALY and increased to $65 \%$ at $20000 € / Q A L Y$.

Conclusions: This economic evaluation shows that stepped-care is dominant to usual care, with a probability of around $60 \%$, due to its clinical superiority and its modest cost savings. However, it depends on the willingness-topay of decision makers whether or not stepped-care is considered cost-effective compared with usual care.

Trial registration: identifier: NTR3296, date: 13-02-2012.

Keywords: Depression, Anxiety, Vision impairment, Stepped-care, Cost-effectiveness, Cost-utility, Economic evaluation

\footnotetext{
* Correspondence: h.vanderaa@vumc.nl

${ }^{1}$ Department of Ophthalmology and the Amsterdam Public Health Research Institute, VU University Medical Centre, De Boelelaan 1117, 1081 Amsterdam, HV, The Netherlands

Full list of author information is available at the end of the article
} 


\section{Background}

Depression and anxiety are highly prevalent in older adults with vision impairment $[1,2]$ and have a negative impact on overall health, vision-related disability, and quality-of-life [3-8]. Moreover, depression and anxiety generate substantial economic burden due to increased healthcare utilization and productivity losses [9-12]. Consequently, vision impairment in old age is a socioeconomic problem. However, research on psychological interventions to treat and prevent mental health problems in this population is scarce $[13,14]$. Moreover, apart from a few studies on the cost-effectiveness of rehabilitation and education to increase wellbeing $[15,16]$, economic evaluation of psychological interventions in the field of low vision is (to our knowledge) completely lacking.

Stepped-care is a model proposed to increase efficiency in mental healthcare. In stepped-care, patients receive subsequent treatment components by order of intensity, i.e. patients start with low-intensity treatments and only move on to higher-intensity treatments when sufficient response is lacking. This care model is expected to lower costs by maximizing the efficiency of resource allocation, and is, therefore, recommended by Dutch and British guidelines (e.g. the National Institute for Health and Clinical Excellence) [17-19]. Several studies outside the field of low vision have shown that stepped-care is cost-effective as compared to usual care in reducing depression and anxiety $[19,20]$. Our group previously showed that stepped-care significantly reduced the incidence of depressive and anxiety disorders in older adults with vision impairment [21]. However, the cost-effectiveness of this intervention has not yet been investigated.

Therefore, the present study aimed to perform a costutility analysis for quality adjusted life years (QALYs) of stepped-care in comparison with usual care and to perform a cost-effectiveness analysis in preventing major depressive and anxiety disorders and reducing symptoms of depression and anxiety in older adults with vision impairment.

\section{Methods}

\section{Design}

An economic evaluation based on a societal perspective was performed alongside a two-armed multicenter randomized controlled trial (RCT), as described in the original protocol [22]. The trial is registered in the Dutch trial registry (identifier: NTR3296, http://www.trialregister.nl). Detailed information on the study design and intervention is provided elsewhere [21, 22].

\section{Participants and setting}

From July 2012 to April 2013, a random sample of 3000 patients of 50 years and older from 17 outpatient clinics of three low vision rehabilitation centers in Belgium and the Netherlands were invited to participate. Of these, $30.5 \%(n=914)$ provided written informed consent, and underwent baseline interviews to determine eligibility.

Patients were eligible if: a) they had subthreshold depression and/or anxiety, i.e. scored $\geq 16$ on the Centre for Epidemiologic Studies Depression scale (CES-D) $[23,24]$ and/or $\geq 8$ on the Hospital Anxiety and Depression Scale-Anxiety subscale (HADS-A) [25, 26], b) they did not meet the DSM-IV criteria of a major depressive, dysthymic and/or anxiety disorder as assessed with the Mini International Neuropsychiatric Interview (MINI) $[27,28], \mathrm{c})$ they adequately spoke the Dutch language, and d) they were not severely cognitively impaired, as assessed with the Six-item screener [29].

\section{Randomization}

A pre-specified power calculation was based on the study of van 't Veer et al. [30] who evaluated the costeffectiveness of stepped-care in the general elderly population. They found an effect size of 0.44. Based on a twosided $\alpha \leq 0.05$, a power of 0.85 , and a dropout rate of $20 \%$, a minimum of 230 patients (115 in each arm) was needed. However, since we observed higher dropout rates than expected at the start of the RCT, more patients were included $(n=265)$.

Participants were randomly assigned to stepped-care plus usual care $(n=131)$ or usual care alone $(n=134)$. An allocation scheme was generated by a computerized random number generator stratified by outpatient clinic and based on blocks of two. After the baseline measurement, an independent researcher performed randomization, which was registered at the low vision rehabilitation center. From the beginning of September 2012 to the end of July 2015, seven telephone interviews (baseline, and after $3,6,9,12,18$ and 24 months) per participant were performed at the VU University Medical Centre in Amsterdam by trained and masked research assistants. Participants were told not to divulge the nature of their treatment. Due to the nature of the stepped-care intervention, therapists and patients could not be masked.

\section{Intervention}

The stepped-care program consisted of four steps that each lasted approximately 3 months The total intervention period lasted 12 months, followed by a 12-month follow-up.

At the end of each 3-month period, elevated levels of depression and/or anxiety (i.e. $\geq 16$ on the CES-D and/or $\geq 8$ on the HADS-A) induced moving on to the following step of the intervention. The first step was a period of watchful waiting, which involved an active decision to not directly treat the depressive and/or anxious symptoms but, instead, to intermittently re-assess these symptoms. In the second step, a guided self-help course based 
on cognitive behavioral therapy (CBT) was given. The course was available in written, digital, audio and Braille formats and was supported by trained occupational therapists. In the third step, problem solving treatment (PST) was offered by trained social workers and psychologists. Finally, when participants still had subthreshold depression and/or anxiety after the third step, they moved to the fourth step, which was a referral to their general practitioner (GP) to discuss other treatment and the use of medication. Participants who developed an actual depressive and/or anxiety disorder as assessed with the MINI, were directly referred to their GP. Usual care for both the stepped-care and usual care group included low vision rehabilitation care and/or care that was offered by other healthcare providers.

\section{Clinical outcomes}

The primary outcomes were QALYs and the cumulative incidence of major depressive, dysthymic and/or anxiety disorders (i.e. panic disorder (without agoraphobia), agoraphobia (without a history of panic disorder), social phobia, and/or generalized anxiety disorder). The latter was assessed at every measurement time point with the Dutch MINI Plus (5.0.0) [27, 28]. QALYs were determined by measuring health-related quality-of-life at baseline and at 12 and 24 months using the EuroQol (EQ-5D-3 L) using the official telephone script; this instrument comprises five dimensions (mobility, self-care, activities of daily living, pain/discomfort and depression/anxiety) with three response options (no problems, some problems, extreme problems) [31]. The EQ-5D-3 L health states were converted to health utility scores using the Dutch tariff, in which 0 corresponds to death and 1 corresponds to full health (range -0.33 to 1 , whereby negative utilities indicate that a health state is valued as worse than death) [31]. With the area under the curve method, QALYs were calculated by multiplying the amount of time a patient spent in a particular health state by the utilities. Changes in utilities between health states were considered to be linear. Secondary outcomes were change in symptoms of depression and anxiety, as assessed with the CES-D and the HADS-A, respectively. The CES-D has 20 items and the HADS-A has 7 items evaluated on a 4-point Likertscale [23-26]. In the cost-effectiveness analyses, the change of symptoms between the start and end of followup was assessed instead of the course of symptoms over time as was done in the effectiveness analysis [21]. This was done to facilitate the interpretation of the incremental cost-effectiveness ratios.

\section{Costs measures}

Costs were collected from a societal perspective (informal care was not included). Healthcare utilization was measured using the self-rated Trimbos and iMTA questionnaire for Costs associated with Psychiatric illness (TiC-P, adapted version) [32] and valued using standard costs from the Dutch costing guideline (Table 1) [33]. Medication was valued using prices from the Royal Dutch Society for Pharmacy. Lost productivity due to absenteeism from paid and unpaid work and presenteeism were measured using the Short Form Health and Labour Questionnaire (SF-HLQ) [34]. Costs of absenteeism from paid work and presenteeism were calculated using mean age and gender-specific income values of the Dutch population and calculated according to the friction method. Compared to the human capital method which assumes any hour not worked as an hour lost, the friction method assumes that after a certain period of time (i.e. 161 days) the sick employee is replaced [35]. Thus, lost productivity costs are generated only during the friction period. Lost productivity costs from unpaid work were valued using a shadow price for informal care $(€ 13.50 / \mathrm{h})$ [33]. Both the TiC-P and SF-HLQ were administered at 6,12,18 and 24 months follow-up with a recall period of 6 months. The costs of the stepped-care program were calculated using a bottom-up approach, i.e. costs were determined by the resources each person used at each step (time, materials, etcetera). Standard costs for occupational therapists, social workers and psychologists obtained from the Dutch costing guideline were used to value these

Table 1 Unit costs to value healthcare utilization

\begin{tabular}{|c|c|c|}
\hline Cost category & Unit & $\begin{array}{l}\text { Unit costs } \\
(2013)^{\mathrm{a}}\end{array}$ \\
\hline General practitioner & Contact & $€ 30.64$ \\
\hline Company physician & Contact & $€ 32.26$ \\
\hline Medical specialist & Contact & $€ 78.33$ \\
\hline Occupational or physiotherapist & Contact & $€ 39.16$ \\
\hline Social worker & Contact & $€ 70.71$ \\
\hline Psychologist or psychiatrist in private practice & Contact & $€ 87.03$ \\
\hline Psychologist or psychiatrist in hospital & Contact & $€ 186.03$ \\
\hline Mental healthcare institute worker & Contact & $€ 186.03$ \\
\hline Alternative healer & Contact & $€ 44.67$ \\
\hline Day treatment for mental care & Day & $€ 167.54$ \\
\hline Admission to regular hospital & Day & $€ 473.23$ \\
\hline Admission to academic hospital & Day & $€ 625.54$ \\
\hline Admission to psychiatric hospital & Day & $€ 252.39$ \\
\hline Admission to rehabilitation center & Day & $€ 369.89$ \\
\hline Admission to nursing home & Day & $€ 258.92$ \\
\hline Admission to other healthcare institution ${ }^{b}$ & Day & $€ 497.17$ \\
\hline Homecare & Hour & $€ 38.07$ \\
\hline Informal care & Hour & $€ 13.50$ \\
\hline
\end{tabular}


resources (see Table 1). The index year was 2013. If necessary, consumer price indices were used to correct prices [36].

\section{Statistical analyses}

First, non-response analyses, dropout analyses, and comparisons of baseline differences between the stepped-care group and usual care group were performed with $\mathrm{X}^{2}$-tests, independent samples $\mathrm{t}$-tests, and non-parametric tests. Second, a cost-effectiveness and cost-utility analysis were performed based on the intention-to-treat principle.

The effectiveness analyses are reported elsewhere [21]. In contrast with the effectiveness analyses, in the present study missing cost and effect data were replaced using multiple imputation with chained equations (MICE) [37]. The number of imputed datasets was increased until the loss of efficiency was less than 5\% resulting in 15 imputed datasets [38]. Variables in the imputation model included all outcome variables, characteristics differing between groups at baseline, variables related to missing data and variables related to the outcome variables. To account for the non-normal distribution of cost data, predictive mean matching was used in the MICE procedure [38]. Results from the multiple datasets were pooled using Rubin's rules [39]. Bivariate regression models were used to estimate cost and effect differences, and incremental cost-effectiveness ratios (ICERs) were calculated. Bias-corrected and accelerated bootstrapping was applied to estimate $95 \%$ confidence intervals $(\mathrm{CI})$ around the mean cost and effect differences (5000 replications). The bootstrapped cost-effect pairs were plotted on a cost-effectiveness (CE) plane for each outcome separately to show the uncertainty around the ICER. The net benefit approach was used to estimate a cost-effectiveness acceptability curve (CEAC) using the pooled cost and effect differences, and the pooled, bootstrapped standard errors. The CEAC shows the probability that stepped-care is cost-effective in comparison with usual care for a range of different ceiling ratios (i.e. the willingness-to-pay for one additional recovered patient), indicating decision uncertainty [40].

For the analyses, SPSS for Windows version 21 (SPSS IBM, New York, USA) and Stata/SE software, version 12 (Stata Corp LP) were used.

\section{Sensitivity analysis}

The primary analysis was based on costs from a societal perspective using the friction method to estimate indirect non-healthcare costs.

In addition, two sensitivity analyses were performed: i) the cost-effectiveness analysis was performed from a healthcare perspective, i.e. including only direct costs, ii) also, to determine productivity losses, the human capital method was used in which every hour not worked is considered as a lost hour.

\section{Results}

Participant flow

Of the 3000 invited participants, responders $(n=914$; $30 \%)$ were significantly younger than non-responders $(n=2086 ; 70 \%)$ (mean difference 4.6 years, $p<0.001$ ). At 24-month follow-up, of the 265 patients eligible/willing to participate, 91 dropped-out (34.3\%; 45 stepped-care and 46 usual care). Participants who dropped-out of the study more often lived in a nursing home and were significantly older than those who did not drop-out $(p<0.05)$. Common reasons for dropout were: mortality, physical or mental inability to continue, or too heavy a burden.

Table 2 presents the baseline characteristics of the two study groups: there was a significant difference in education level between the groups $(p<0.05)$.

\section{Clinical outcomes}

The cumulative incidence of depressive/anxiety disorders at 24-month follow-up was 0.29 in the stepped-care group and 0.46 in the usual care group. The absolute risk reduction was 0.17 ; this difference was significant (95\% CI 0.06 to 0.29 ) (Table 3). In addition, imputed and pooled outcomes showed a significant difference between the stepped-care group and usual care group for the HADS-A (mean difference 1.43 , 95\% CI 0.10 to 2.77 ), and a nonsignificant difference for the CES-D (mean difference 2.73, 95\% CI -0.28 to 5.74 ) and QALYs (mean difference 0.03 , $95 \%$ CI -0.09 to 0.15$)$. Note that these latter analyses are different from those performed in our earlier study. ${ }^{21}$

\section{Costs}

All participants in the stepped-care group received watchful waiting $(n=131), 56 \%$ received CBT-based guided self-help, 22\% received PST, and 5\% were referred to their GP as part of the last step of the intervention.

Cost and effect data are presented in Table 3. The mean total intervention costs amounted to $€ 262$ per participant. Although direct healthcare costs were lower for the stepped-care group compared with the usual care group, the mean difference was not significant (-€1154; 95\% CI -7708 to 4328 ). Cost savings were mainly due to significantly lower secondary mental healthcare and hospitalization costs. Indirect non-healthcare costs based on the friction method were not significantly higher for the stepped-care group compared with the usual care group ( $€ 277 ; 95 \%$ CI -1418 to 2230 ). Total costs were lower for the stepped-care group compared to usual care (-€877; 95\% CI -8039 to 5489 ) but the difference was not significant.

\section{Cost utility and cost-effectiveness}

The cost-utility analysis resulted in an ICER of $-29,233$ indicating that to gain 1 QALY $€ 29,233$ is saved in the stepped-care group as compared to usual care. The CE 
Table 2 Baseline patient characteristics for the stepped-care $(n=131)$ and usual care group $(n=134)$

\begin{tabular}{|c|c|c|c|}
\hline \multicolumn{2}{|c|}{ Patient characteristics measured at baseline } & Stepped-care $(n=131)$ & Usual care $(n=134)$ \\
\hline \multicolumn{2}{|l|}{ Gender (female) (n (\%)) } & $91(69 \%)$ & $94(70 \%)$ \\
\hline \multicolumn{2}{|c|}{ Age (years) range [50-98] (mean (SD)) } & $72.4(12.5)$ & $74.9(11.9)$ \\
\hline \multicolumn{2}{|c|}{ Education (years) range [0-16] (mean (SD)) } & $10.4(3.8)$ & $9.3(3.4)$ \\
\hline \multicolumn{2}{|l|}{ Having work (n (\%)) } & $15(12 \%)$ & $7(5 \%)$ \\
\hline \multirow[t]{3}{*}{ Nationality (n (\%)) } & Dutch & $116(89 \%)$ & $117(87 \%)$ \\
\hline & Belgian & $14(11 \%)$ & $16(12 \%)$ \\
\hline & Other & $1(1 \%)$ & $1(1 \%)$ \\
\hline \multicolumn{2}{|c|}{ Living situation (independent) (n (\%)) } & $115(88 \%)$ & $124(93 \%)$ \\
\hline \multirow[t]{3}{*}{ Income (n (\%)) } & Usually enough money & $61(47 \%)$ & $62(46 \%)$ \\
\hline & Just enough money & $55(42 \%)$ & $57(43 \%)$ \\
\hline & Not enough money & $10(8 \%)$ & $15(11 \%)$ \\
\hline \multirow[t]{6}{*}{ Cause of vision loss (n (\%)) } & Macular degeneration & $62(47 \%)$ & $60(45 \%)$ \\
\hline & Glaucoma & $26(20 \%)$ & $19(14 \%)$ \\
\hline & Cataract & $26(20 \%)$ & $19(14 \%)$ \\
\hline & Diabetic retinopathy & $5(4 \%)$ & $4(3 \%)$ \\
\hline & Cerebral hemorrhage & $5(4 \%)$ & $10(8 \%)$ \\
\hline & Other & $45(34 \%)$ & $60(45 \%)$ \\
\hline \multicolumn{2}{|c|}{ Time of onset (years) range [0-79] (mean (SD)) } & $16.0(19.6)$ & $14.4(18.2)$ \\
\hline \multirow[t]{3}{*}{ LogMAR visual acuity (n (\%)) } & Normal visual acuity & $9(7 \%)$ & $15(11 \%)$ \\
\hline & Mild vision loss & $24(18 \%)$ & $23(17 \%)$ \\
\hline & Low vision / blindness & $86(66 \%)$ & $86(64 \%)$ \\
\hline \multicolumn{2}{|c|}{ Comorbidity range [0-5] (mean (SD)) } & $1.1(1.2)$ & $1.2(1.2)$ \\
\hline \multicolumn{2}{|c|}{ History of major depressive disorder (n (\%)) } & $30(23 \%)$ & $25(19 \%)$ \\
\hline \multicolumn{2}{|c|}{ History of dysthymic disorder (n (\%)) } & $4(3 \%)$ & $1(1 \%)$ \\
\hline \multicolumn{2}{|l|}{ History of panic disorder (n (\%)) } & $8(6 \%)$ & $8(6 \%)$ \\
\hline \multicolumn{2}{|l|}{ Baseline CES-D score (mean(SD)) } & $21.2(6.4)$ & $21.1(6.7)$ \\
\hline \multicolumn{2}{|c|}{ Baseline HADS-A score (mean(SD)) } & $7.1(4.1)$ & $7.1(3.8)$ \\
\hline \multicolumn{2}{|c|}{ Baseline EQ-5D utility score (mean(SD)) } & $0.7(0.3)$ & $0.7(0.2)$ \\
\hline
\end{tabular}

SD standard deviation, CES-D Center for Epidemiologic Studies Depression, HADS-A Hospital Anxiety and Depression Scale-Anxiety, EQ-5D Euroqol-5 Dimensions

plane and the CEAC in Fig. 1 a and b show that the probability that stepped-care was cost-effective compared to usual care was $59 \%$ or more for a ceiling ratio of $€ 0$ per QALY and that this increased to $65 \%$ or more at a willingness-to-pay of $€ 20,000$ per QALY.

With regard to the cumulative incidence of depressive/ anxiety disorders, the ICER was -5159 indicating that to prevent one case of depression or anxiety $€ 5159$ is saved in the stepped-care group as compared to usual care (Table 4, Fig. 1). The CE plane and the CEAC in Fig. $1 \mathrm{c}$ and $\mathrm{d}$ show that, for a ceiling ratio of $€ 0$ per disorder prevented, the probability that stepped-care was cost-effective compared to usual care was $59 \%$. At a willingness-to-pay of $€ 10,000$ this probability was $77 \%$, and at a willingnessto-pay of $€ 20,000$ this probability was $88 \%$. The probability of cost-effectiveness increased to $95 \%$ or more at a willingness-to-pay of $€ 33,000$ per disorder prevented.
For the CES-D and the HADS-A, cost-effectiveness acceptability curves show that the probability of costeffectiveness was $60 \%$ for both outcomes for a ceiling ratio of $€ 0$ per point improvement on the CES-D/HADSA; this probability increased to $95 \%$ or more at a willingness-to-pay of $€ 2500$ per point improvement on the CES-D and $€ 4000$ per point improvement on the HADS-A (Table 4).

\section{Sensitivity analysis}

Based on the societal perspective with the human capital method (non-significant) higher total costs were found for the stepped-care group compared to the usual care group (mean difference 200, 95\% CI -7035 to 6829) (Table 4). The cost-effectiveness acceptability curves show that the probability of cost-effectiveness was $47 \%$ or more for a ceiling ratio of $€ 0$ per disorder prevented and that this increased to 
Table 3 Multiple-imputed effects and $\operatorname{costs}^{\mathrm{a}}$ for the stepped-care $(n=131)$ and usual care group $(n=134)$ after 24 months

\begin{tabular}{|c|c|c|c|c|}
\hline \multicolumn{2}{|l|}{ Outcome } & $\begin{array}{l}\text { Stepped-care }(n=131) \\
(\text { mean }(\mathrm{SE}))\end{array}$ & $\begin{array}{l}\text { Usual care }(n=134) \\
\text { (mean }(\mathrm{SE}))\end{array}$ & $\begin{array}{l}\text { Mean difference } \\
(95 \% \mathrm{Cl})^{\mathrm{b}}\end{array}$ \\
\hline \multicolumn{2}{|c|}{ Cumulative incidence of depressive/anxiety disorders } & $0.29(0.04)$ & $0.46(0.04)$ & 0.17 (0.06 to 0.29$)$ \\
\hline \multicolumn{2}{|c|}{ Mean change CES-D score } & $6.40(1.05)$ & $3.67(0.99)$ & $2.73(-0.28$ to 5.74$)$ \\
\hline \multicolumn{2}{|c|}{ Mean change HADS-A score } & $1.88(0.47)$ & $0.45(0.51)$ & $1.43(0.10$ to 2.77$)$ \\
\hline \multicolumn{2}{|l|}{ QALY } & $1.32(0.04)$ & $1.28(0.04)$ & $0.03(-0.09$ to 0.15$)$ \\
\hline \multirow[t]{5}{*}{ Direct healthcare costs } & Medication costs & $1705(245)$ & $1783(419)$ & $-78(-938$ to 505$)$ \\
\hline & Primary care & $10,911(1496)$ & $10,124(1631)$ & 787 (-3754 to 4910) \\
\hline & Secondary care & $3783(675)$ & $5909(1456)$ & $-2126(-5911$ to 348$)$ \\
\hline & Intervention costs & $262(34)$ & $0(0)$ & 262 (204 to 340) \\
\hline & Total & $16,661(1691)$ & $17,815(2680)$ & $-1154(-7708$ to 4328$)$ \\
\hline \multicolumn{2}{|c|}{ Indirect non healthcare costs ${ }^{c}$} & $5270(771)$ & $4993(583)$ & 277 (-1418 to 2230$)$ \\
\hline \multicolumn{2}{|l|}{ Total costs } & 21,931 (2035) & $22,808(2956)$ & -877 (-8039 to 5489$)$ \\
\hline
\end{tabular}

${ }^{\mathrm{a}}$ Costs are presented in $€$ and indexed to the year 2013

${ }^{\mathrm{b}}$ For cost measures bootstrapped $95 \%$ confidence intervals were used

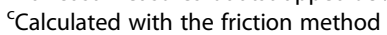

SE standard error, Cl confidence interval, CES-D Center for Epidemiologic Studies Depression, HADS-A Hospital Anxiety and Depression Scale-Anxiety, QALY quality adjusted life year

$95 \%$ or more at a willingness-to-pay of $€ 42,500$ per disorder prevented. Based on the healthcare perspective, compared with the main analysis a larger cost difference was found, with stepped-care being less costly than usual care (mean difference $-1154,95 \%$ CI -7708 to 4328 ) (Table 4). The cost-effectiveness acceptability curves show that the probability of cost-effectiveness was $59 \%$ or more for a ceiling ratio of $€ 0$ per disorder prevented and that this increased to $95 \%$ or more at a willingness-to-pay of $€ 26,000$ per disorder prevented (see Additional file 1: Figure S1 with CE-planes and CEACs).

\section{Discussion}

This economic evaluation shows that stepped-care is dominant to usual care, with a probability of around 60\%, due to its clinical superiority and its modest (non-significant) cost savings, mainly due to lower secondary mental healthcare use and hospitalization, as compared to usual care. Mean intervention costs are low (i.e. €262 per participant) and comparable to other stepped-care programs $[20,41]$. Based on the cost-effectiveness acceptability curves, it is shown that the probability that the intervention is cost-effective compared to usual care is $95 \%$ or more when society is willing to pay $€ 33,000$ per disorder prevented. Lost productivity costs were higher for stepped-care compared to usual care. However, because in this older population only a few participants had a paid job (8\%), the analysis was also performed from a healthcare perspective (including only direct healthcare costs); this showed slightly more positive outcomes. Based on this perspective there is a $95 \%$ probability of stepped-care being cost-effective compared to usual care when decision makers are willing to pay $€ 26,000$ per disorder prevented.
Although lower than in the main analysis, this amount is still relatively large and decision makers have to decide whether this is acceptable.

Although stepped-care was significantly more effective in preventing depressive and anxiety disorders compared with usual care, the difference in QALYs, in favor of stepped-care, was not statistically significant. QALY is a measure of health-related quality-of-life (measured with the EQ-5D in this study), including various dimensions of which mental health is covered in only one dimension. When analyzing the dimensions separately, stepped-care significantly reduced problems with activities of daily living and depression/anxiety compared with usual care; however, no significant difference was found on the other dimensions (i.e., mobility, self-care, pain/discomfort) (data not shown). The lack of an overall significant effect might be explained by the nature of the outcome, i.e. because stepped-care was specifically aimed at improving mental health, similar improvements in overall quality-of-life cannot be expected. In addition, the impact of vision loss itself on different elements of health-related quality of life is likely to influence overall quality of life in this specific population, but these effects cannot be separated. Moreover, the EQ-5D with threelevel response options has limited ability to distinguish small to moderate differences in health status [42].

\section{Strengths and limitations}

A strength of this study is the pragmatic design that was chosen. This helps policymakers make evidence-based decisions on whether implementation of stepped-care can be considered an efficient allocation of scarce resources in real-life situations and to increase 

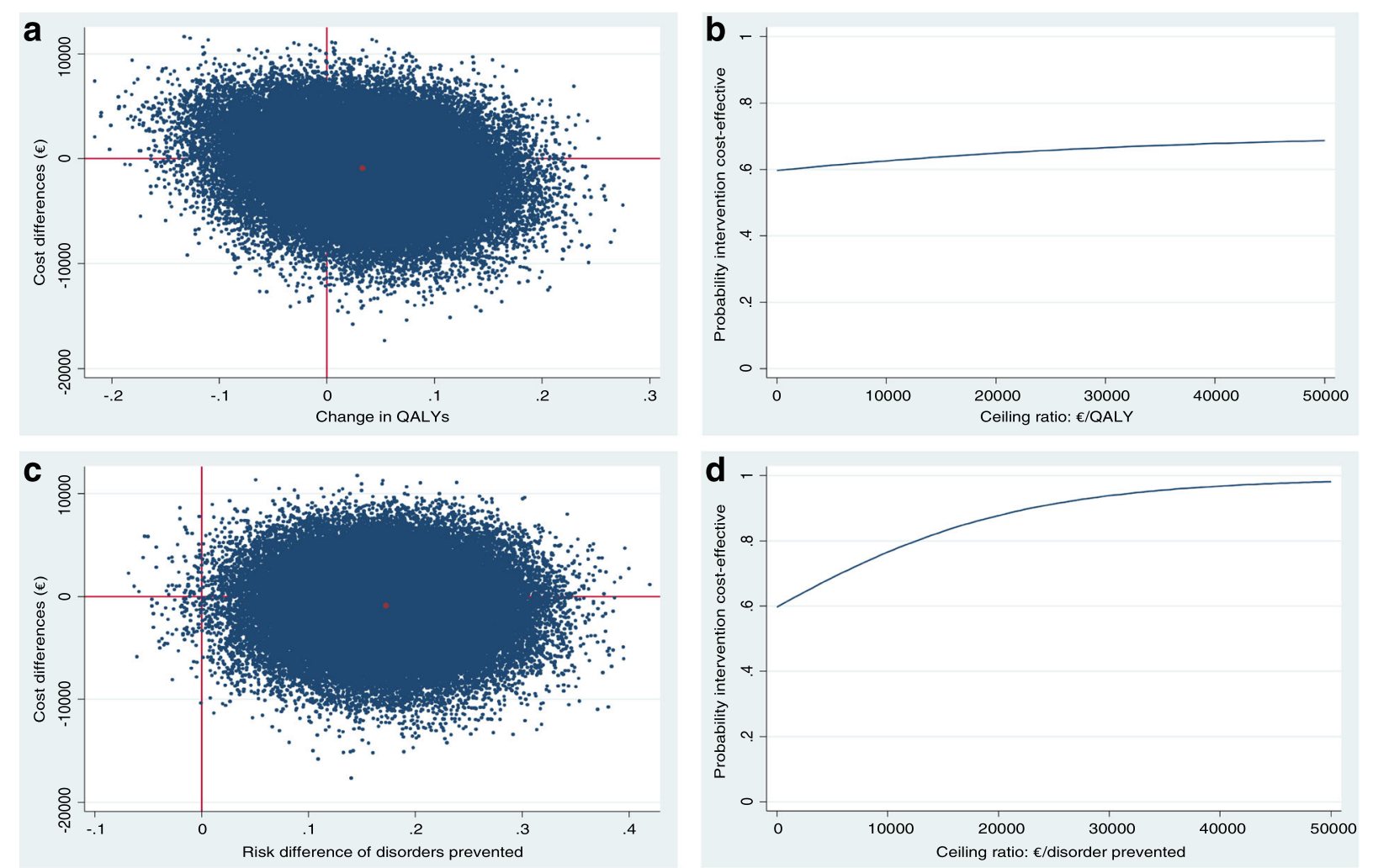

Fig. 1 a Cost-effectiveness plane. Showing the change in quality adjusted life years (QALY) during 24 months follow-up in the stepped-care versus the usual care group from a societal perspective using the friction method. The red dot indicates the point estimate of the ICER, mean difference was 0.03 and $€ 877$ less costs were made in the stepped-care group. The grey dots indicate the bootstrapped cost-effects pairs reflecting the uncertainty surrounding the ICER. $\mathbf{b}$ Cost-effectiveness acceptability curve. Showing the probability that the stepped-care intervention is cost-effective compared to the control condition from a societal perspective using the friction method in change in quality adjusted life-years (QALY) over a range of values for the maximum acceptable ceiling ratio. c Cost-effectiveness plane. Showing the percentage of major depressive and anxiety disorders prevented during 24 months follow-up in the stepped-care versus the usual care group from a societal perspective using the friction method. The red dot indicates the point estimate of the incremental cost-effectiveness ratio (ICER, 17\% of disorders were prevented and €877 less costs were made in the stepped-care group). The grey dots indicate the bootstrapped cost-effects pairs reflecting the uncertainty surrounding the ICER. $\mathbf{d}$ Cost-effectiveness acceptability curve. Showing the probability that the stepped-care intervention is cost-effective compared to the control condition from a societal

perspective using the friction method in preventing major depressive and anxiety disorders over a range of values for the maximum acceptable ceiling ratio

generalizability of the results. Second, a long follow-up period was chosen to assess long-term treatment effects. Third, treatment arms appeared to be well randomized with no relevant differences in baseline characteristics between the stepped-care and usual care group. A limitation of this study is the high dropout rate: $34 \%$ in total. However, there was no significant difference in dropout between the stepped-care and usual care group and there was sufficient statistical power. Missing data were dealt with by applying multiple imputation techniques, which is the preferred method in economic evaluations [43]. Second, the relatively large recall periods for healthcare utilization and work productivity (6 months) may have introduced recall bias. Third, informal care was not included in the total costs from a societal perspective. However, it is hard to estimate how inclusion of informal care costs would have influenced our societal cost estimates. Fourth, although the pragmatic design of our study increases generalizability and the outcomes could be used for stepped-care in different settings, strictly speaking our outcomes can only be generalized to visually impaired older adults who are registered at a low vision rehabilitation organization.

\section{Implications for practice and future research}

This study shows that stepped-care is dominant to usual care (with a probability of around 60\%) in treating mental health problems in visually impaired older adults. Stepped-care enables professionals to efficiently deploy their limited resources by initially offering low intensity and low cost interventions and only moving on to higher-intensity and more costly interventions when sufficient response is lacking. The stepped-care program is effective in preventing major depressive/anxiety disorders as compared to usual care; the probability that stepped-care is cost-effective compared to usual care is 
Table 4 Results of the cost-effectiveness and cost-utility analyses

\begin{tabular}{|c|c|c|c|c|c|c|c|c|}
\hline \multirow[t]{2}{*}{ Outcome } & \multirow[t]{2}{*}{ Analysis } & \multirow{2}{*}{$\begin{array}{l}\text { Mean cost difference } \\
\text { (stepped-care - usual care } \\
\text { (bootstrapped } 95 \% \text { CI)) }\end{array}$} & \multirow{2}{*}{$\begin{array}{l}\text { Mean change difference } \\
\text { (stepped-care - usual care } \\
(95 \% \mathrm{Cl}) \text { ) }\end{array}$} & \multirow[t]{2}{*}{ ICER } & \multicolumn{4}{|c|}{ CE-plane ${ }^{a}$} \\
\hline & & & & & $\overline{\mathrm{NE}}$ & SE & SW & $\overline{N W}$ \\
\hline \multirow[t]{3}{*}{ Disorder } & Societal perspective (friction) & -877 (-8039 to 5489$)$ & \multirow[t]{3}{*}{0.17 (0.06 to 0.29$)$} & -5159 & $41 \%$ & $59 \%$ & $0 \%$ & $0 \%$ \\
\hline & Societal perspective (human capital) & $200(-7035$ to 6829) & & 1176 & $53 \%$ & $47 \%$ & $0 \%$ & $0 \%$ \\
\hline & Healthcare perspective & -1154 ( -7708 to 4328$)$ & & -6788 & $37 \%$ & $63 \%$ & $0 \%$ & $0 \%$ \\
\hline \multirow[t]{3}{*}{ CES-D } & Societal perspective (friction) & -877 ( -8039 to 5489$)$ & \multirow[t]{3}{*}{$2.73(-0.28$ to 5.74$)$} & -321 & $39 \%$ & $58 \%$ & $2 \%$ & $1 \%$ \\
\hline & Societal perspective (human capital) & $200(-7035$ to 6829$)$ & & 73 & $51 \%$ & $46 \%$ & $1 \%$ & $2 \%$ \\
\hline & Healthcare perspective & -1154 ( -7708 to 4328$)$ & & -423 & $35 \%$ & $62 \%$ & $2 \%$ & $1 \%$ \\
\hline \multirow[t]{3}{*}{ HADS-A } & Societal perspective (friction)) & -877 (-8039 to 5489$)$ & \multirow[t]{3}{*}{$1.43(0.10$ to 2.77$)$} & -613 & $40 \%$ & $58 \%$ & $2 \%$ & $0 \%$ \\
\hline & Societal perspective (human capital & $200(-7035$ to 6829$)$ & & 140 & $52 \%$ & $46 \%$ & $1 \%$ & $1 \%$ \\
\hline & Healthcare perspective & -1154 (-7708 to 4328) & & -807 & $36 \%$ & $63 \%$ & $1 \%$ & $0 \%$ \\
\hline \multirow[t]{3}{*}{ QALY } & Societal perspective (friction) & -877 ( -8039 to 5489$)$ & \multirow[t]{3}{*}{$0.03(-0.09$ to 0.15$)$} & $-29,233$ & $25 \%$ & $45 \%$ & $14 \%$ & $16 \%$ \\
\hline & Societal perspective (human capital) & $200(-7035$ to 6829$)$ & & 66,667 & $34 \%$ & $36 \%$ & $11 \%$ & $19 \%$ \\
\hline & Healthcare perspective & $-1154(-7708$ to 4328$)$ & & $-38,467$ & $23 \%$ & $48 \%$ & $16 \%$ & $13 \%$ \\
\hline
\end{tabular}

Cost-effectiveness and cost-utility analyses were based on: 1) societal perspective and the friction method, 2) societal perspective and the human capital method, and 3) healthcare perspective. Cl confidence interval, CES-D Center for Epidemiologic Studies Depression, HADS-A Hospital Anxiety and Depression Scale-Anxiety, QALY quality adjusted life year, ICER incremental cost-effectiveness ratio, CE cost-effectiveness, NE north-east quadrant, SE south-east quadrant, SW south-west quadrant, NW north-west quadrant. ${ }^{\text {a}}$ Effect estimates for Disorder, CES-D and HADS-A were multiplied by -1 in the CE plane to maintain the usual meaning of the quadrants

$95 \%$ or more at a willingness-to-pay of $€ 33,000$ per disorder prevented. Future studies should investigate how the cost-effectiveness of stepped-care can be improved. Possible options for this are: offering interventions tailored to personal needs and symptom severity, e.g. varying the watchful waiting period [44], and directly offering higher intensity interventions to persons with a history of depressive/anxiety disorder [21]. In addition, other evidence-based treatment options (e.g. exercise programs, e-mental health) could be added to the model. Moreover, the present study, which investigates both the clinical effectiveness and cost-effectiveness of a promising intervention, could serve as an example for other intervention studies in the field of low vision. Considering that vision impairment is ubiquitous in an aging population and that resources in healthcare are scarce, such economic evaluations are highly relevant.

\section{Additional file}

Additional file 1: Figure S1. Cost-effectiveness planes and costefectiveness acceptability curves for the sensitivity analyses. (DOCX $56 \mathrm{~kb}$ )

\section{Abbreviations}

CBT: Cognitive behavioral therapy; CE: Cost-effectiveness; CEAC: Cost-effectiveness acceptability curve; CES-D: Centre for Epidemiologic Studies Depression scale; Cl: Confidence interval; EQ-5D: EuroQol 5-Dimensions; GP: General practitioner; HADS-A: Hospital Anxiety Depression Scale - Anxiety; ICER: Incremental cost-effectiveness ratios; iMTA: Questionnaire for Costs associated with Psychiatric illness; MICE: Multiple imputation techniques with chained equations; MINI: Mini International Neuropsychiatric Interview; PST: problem solving treatment; QALY: Quality adjusted life year; RCT: Randomized controlled trial; SF-HLQ: Short Form Health and Labor Questionnaire; TiC-P: Trimbos

\section{Acknowledgements}

The authors extend special thanks to Sie Pei Lim (MSc) who, during her internship at the VU University Medical Center Amsterdam, had an important role in preparing the data for this manuscript.

\section{Funding}

The study was supported by 'ZonMw InZicht', the Dutch Organization for Health Research and Development - InSight Society [grant number 60-0063598108]. The sponsor had no role in the design or conduct of this research.

\section{Availability of data and materials}

Full dataset and statistical code is available from the corresponding author on reasonable request.

\section{Authors' contributions}

The executive researcher ( $H v d A)$, project leader (GvR), clinical psychologist, advisor $(\mathrm{HC})$, health economics advisor (JB), and principal investigator (RvN) conceived the study and its design. A plan for the statistical analysis was developed by JB and HvdA. Data was analyzed and interpreted by HvdA, with support from JB. HvdA and RvN had full access to all the data in the study and take responsibility for the integrity of the data and the accuracy of the data analysis. HvdA drafted the manuscript which was revised by the other authors. All authors read and approved the final manuscript.

\section{Ethics approval and consent to participate}

The study was conducted according to the principles of the Declaration of Helsinki, and approved by the Medical Ethics Committee of the University Hospital Leuven and the VU University Medical Centre Amsterdam. All participants provided written informed consent after explanation of the nature and possible consequences of the study.

\section{Consent for publication}

Not applicable.

\section{Competing interests}

The authors declare that they have no competing interests.

\section{Publisher's Note}

Springer Nature remains neutral with regard to jurisdictional claims in published maps and institutional affiliations. 


\section{Author details}

'Department of Ophthalmology and the Amsterdam Public Health Research Institute, VU University Medical Centre, De Boelelaan 1117, 1081 Amsterdam, HV, The Netherlands. ${ }^{2}$ Department of Ophthalmology, Elkerliek Hospital, Wesselmanlaan 25, 5707 Helmond, HA, The Netherlands. ${ }^{3}$ Department of Health Sciences and the Amsterdam Public Health Research Institute, Faculty of Earth and Life Sciences, VU University Amsterdam, De Boelelaan 1105, 1081 Amsterdam, HV, The Netherlands. ${ }^{4}$ Department of Psychiatry VUmc/ GGZinGeest, A.J.Ernststraat 1187, 1081 Amsterdam, HL, The Netherlands.

Received: 6 December 2016 Accepted: 20 July 2017

\section{Published online: 01 August 2017}

\section{References}

1. Pascolini D, Mariotti SP. Global estimates of visual impairment: $2010 . \mathrm{Br}$ Ophthalmol. 2012;96:614-8.

2. Van der Aa HP, Comijs HC, Penninx BW, van Rens GH, van Nispen RM. Major depressive and anxiety disorders in visually impaired older adults. Invest Ophth Vis Sci. 2015;56:849-54.

3. Beekman AT, Copeland JR, Prince MJ. Review of community prevalence of depression in later life. Br J Psychiatry. 1999;174:307-11.

4. Bryant $\mathrm{C}$, Jackson $\mathrm{H}$, Ames $\mathrm{D}$. The prevalence of anxiety in older adults: methodological issues and a review of the literature. J Affect Disord. 2008;109:233-50.

5. Casten R, Rovner B. Update on depression in age-related macular degeneration. Curr Opin Ophthalmol. 2013;24:239-43.

6. Jones GC, Rovner BW, Crews JE, Danielson ML. Effects of depressive symptoms on health behaviour practices among older adults with vision loss. Rehabil Psychol. 2009;54:164-72.

7. Rovner BW, Casten RJ, Hegel MT, Tasman WS. Minimal depression and vision function in age-related macular degeneration. Ophthalmol. 2006;113:1743-7.

8. Adamson JA, Price GM, Breeze E, Bulpitt CJ, Fletcher AE. Are older people dying of depression? Findings from the Medical Research Council trial of the assessment and management of older people in the community. J Am Geriatr Soc. 2005:53:1128-32.

9. Smit F, Everdeen A, Cuijpers P, et al. Opportunities for cost-effective prevention of late life depression: an epidemiological approach. Arch Gen Psychiatry. 2006;63:290-6.

10. Cuijpers P, Smit F, Oostenbrink J, De Graaf R, Ten Have M, Beekman A. Economic costs of minor depression: a population-based study. Acta Psychiatr Scand. 2007;115:229-36.

11. Konnopka A, Leichsenring F, Leibing E, König HH. Cost-of-illness studies and cost- effectiveness analyses in anxiety disorders: a systematic review. J Affect Disord. 2009:114:14-31.

12. Luppa M, Heinrich S, Angermeyer MC, König HH, Riedel-Heller SG. Cost-ofillness studies of depression: a systematic review. J Affect Disord. 2007:98:29-43.

13. Van der Aa HPA, Margrain TH, Van Rens GHMB, Heymans MW, Van Nispen RMA. Psychosocial interventions to improve mental health in adults with vision impairment: systematic review and meta-analysis. Ophthalmic Physiol Opt. 2016:36:584-606.

14. Binns AM, Bunce C, Dickinson C, et al. How effective is low vision service provision? A Systematic Review Survey of Ophthalmology. 2012;51:34-65.

15. Eklund $K$, Sonn U, Nystedt P, Dahlin-Ivanoff S. A cost-effectiveness analysis of a health education programme for elderly persons with age-related maculardegeneration: a longitudinal study. Disabil Rehabil. 2005;27:1203-12.

16. Stroupe KT, Stelmack JA, Tang XC, Reda DJ, Moran D, Rinne S, Mancil R, Wei Y, Cummings R, Mancil G, Ellis N, Massof RW. Econmic evaulation of blind rehabilitation for veterans with macular diseases in the department of veterans affairs. Ophthalmic Epidemiol. 2008:15:84-91.

17. National Institute for Health and Clinical Excellence (NICE). Depression in adults with a chronic physical health problem. http://www.nice.org.uk/ guidance/cg91 (accessed 5 December 2014).

18. Hutschemaekers GJM. Multidisciplinary guidelines in Dutch mental healthcare: plans, bottlenecks and possible solutions. Int J Integr Care. 2003;3:e10.

19. van Straten A, Hill J, Richards DA, Cuijpers P. Stepped-care treatment delivery for depression: a systematic review and meta-analysis. Psychol Med. 2014;45:231-46.

20. Van't Veer-Tazelaar $P$, Smit F, van Hout H, van Oppen $P$, van der Horst $H_{\text {, }}$ Beekman A, van Marwijk H. Cost-effectiveness of a stepped-care intervention to prevent depression and anxiety in late life: randomised trial. Br J Psychiatry. 2010;196:319-25.
21. van der Aa HP, van Rens GH, Comijs HC, Margrain TH, Galindo-Garre F, Twisk JW, van Nispen RM. Stepped-care for depression and anxiety in visually impaired older adults: multicentre randomised controlled trial. BMJ. 2015;315:h6127.

22. van der $\mathrm{Aa} \mathrm{HP}$, van Rens $\mathrm{GH}$, Comijs HC, et al. Stepped-care to prevent depression and anxiety in visually impaired older adults - design of a randomised controlled trial. BMC Psychiatry. 2013;13:209.

23. Beekman AT, Deeg DJ, van Limbeek J, Braam AW, De Vries MZ, van Tilburg W. Criterion validity of the Centre for Epidemiologic Studies Depression scale (CES-D): results from a community-based sample of older subjects in The Netherlands. Psychol Med. 1997;27:231-5.

24. Breslau N. Depressive symptoms, major depression, and generalized anxiety: a comparison of self-reports on CES-D and results from diagnostic interviews. Psychiatry Res. 1985;15:219-29.

25. Mykletun A, Stordal E, Dahl AA. Hospital anxiety and depression (HAD) scale: factor structure, item analyses and internal consistency in a large population. Br J Psychiatry. 2001;179:540-4.

26. Bjelland I, Dahl AA, Haug TT, Neckelmann D. The validity of the hospital anxiety and depression scale. An updated literature review. J Psychosom Res. 2002;52:69-77.

27. Sheehan DV, Lecrubier $Y$, Sheehan $\mathrm{KH}$, et al. The Mini-international neuropsychiatric interview (M.I.N.I.): the development and validation of a structured diagnostic psychiatric interview for DSM-IV and ICD-10. J Clin Psychiatry. 1998:59:22-33.

28. van Vliet IM, de Beurs E. The MINI-international neuropsychiatric interview. A brief structured diagnostic psychiatric interview for DSM-IV and ICD-10 psychiatric disorders. Tijdschr Psychiatr. 2007:49:393-7.

29. Callahan CM, Unverzagt FW, Hui SL, Perkins AJ, Hendrie HC. Six-item screener to identify cognitive impairment among potential subjects for clinical research. Med Care. 2002;40:771-81.

30. van't Veer-Tazelaar PJ, van Marwijk HWJ, van Oppen P, et al. Stepped-care prevention of anxiety and depression in late life: a randomized controlled trial. Arch Gen Psychiatry. 2009;66:297-304.

31. Lamers LM, Stalmeier PF, McDonnell J, Krabbe PF, van Busschbach JJ. Measuring the quality of life in economic evaluations: the Dutch EQ-5D tariff. Ned Tijdschr Geneeskd. 2005:149:1574-8.

32. Hakkaart-van Roijen L. Manual Trimbos/iMTA questionnaire for costs associated with psychiatric illness (TiC-P). Institute for Medical Technology Assessment. Rotterdam: Erasmus University Medical Centre; 2002.

33. Hakkaart-van Roijen L, Tan SS, Bouwmans CAM: Dutch manual for costing in economic evaluations. Diemen: College Voor Zorgverzekeringen (CVZ); 2011.

34. Hakkaart-van Roijen L, CAM B. Manual Short Form-Health and Labour Questionnaire (SF-HLQ). 07.103. In: Rotterdam. Institute for Medical Technology Assessment: Erasmus University Medical Center; 2007.

35. Brouwer WB, Koopmanschap MA. The friction-cost method : replacement for nothing and leisure for free? PharmacoEconomics. 2005;23:105-11.

36. Statistics Netherlands (2012) Voorburg/Heerlen: Centraal Bureau voor de Statistiek (CBS).

37. van Buuren S, Groothuis-Oudshoorn K. MICE: multivariate imputation by chained equations in R. J Stat Softw. 2011:45:1-68.

38. White IR, Royston P, Wood AM. Multiple imputation using chained equations: issues and guidance for practice. Stat Med. 2011 Feb 20;30(4):377-99.

39. Rubin DB. Multiple imputation for nonresponse in surveys. New York: John Wiley \& Sons; 1987.

40. Fenwick E, O'Brien BJ, Briggs A. Cost-effectiveness acceptability curves-facts, fallacies and frequently asked questions. Health Econ. 2004;13:405-15.

41. Bosmans JE, Dozeman E, van Marwijk HW, van Schaik DJ, Stek ML, Beekman AT, van der Horst HE. Cost-effectiveness of a stepped-care programme to prevent depression and anxiety in residents in homes for the older people: a randomised controlled trial. Int J Geriatr Psychiatry. 2014:29:182-90.

42. Janssen MF, Pickard AS, Golicki D, et al. Measurement properties of the EQ5D-5L compared to the EQ-5D-3L across eight patient groups: a multicountry study. Qual Life Res. 2013;22:1717-27.

43. Burton A, Billingham $\sqcup$, Bryan S. Multiple imputation is the preferred method to estimate missing data in economic evaluations. Clin Trials. 2007:4:154-61.

44. van der Aa HP, Krijnen-de Bruin E, van Rens GH, Twisk JW, van Nispen RM. Watchful waiting for subthreshold depression and anxiety in visually impaired older adults. Qual Life Res. 2015;24:2885-93. 